\title{
COST-BASED DECISION MAKING MODEL FOR REGIONAL IN-HOUSE VERSUS OUTSOURCING LOGISTICS
}

\author{
A Thesis \\ presented to \\ the Faculty of California Polytechnic State University, \\ San Luis Obispo
}

\author{
In Partial Fulfillment \\ of the Requirements for the Degree \\ Master of Industrial Engineering
}

by

Henrik Darren Lee

December 2015 
(C) 2015

Henrik Darren Lee

ALL RIGHTS RESERVED 


\section{COMMITTEE MEMBERSHIP}

TITLE:

Cost-based decision making model for regional in-house versus outsourcing logistics

AUTHOR: Henrik Darren Lee

DATE SUBMITTED: December 2015

COMMITTEE CHAIR: $\quad$ Tali Freed, Ph.D.

Professor of Industrial/Manufacturing Engineering

COMMITTEE MEMBER: Beth Chance, Ph.D.

Professor of Statistics

COMMITTEE MEMBER: Liz Schlemer, Ph.D.

Professor of Industrial/Manufacturing Engineering 


\begin{abstract}
Cost-based decision making model for regional in-house versus outsourcing logistics Henrik Darren Lee
\end{abstract}

This thesis proposes a model for selecting between insourcing logistics for local distribution and outsourcing these functions to a third-party logistics (3PL) company. Supply chain optimization, as well as global supply chain management, are topics that are now well-covered; local distribution, despite its integral function in an organization, is much less researched. Models exist for network design and optimization, but the practical application of these network models may call for decisions and considerations that are not covered in these optimization models.

A breakeven analysis selection model between using in-house logistic system and 3PL is derived, considering various regional parameters. The model is subsequently tested with sample parameters. Using this system as a basis, the thesis then moves on to analyze the potential of employing in-house logistics where, based on client density, outsourcing to $3 \mathrm{PL}$ is initially thought to be more cost-efficient.

The proposed model can be used for the evaluation and selection of logistics systems. In addition, the model can be used for decision making regarding inventory decentralization. 


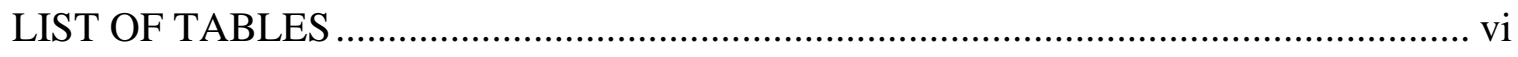

LIST OF FIGURES ……….............................................................................. vii

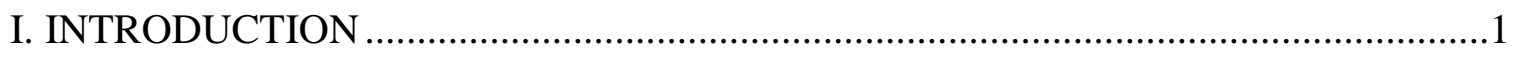

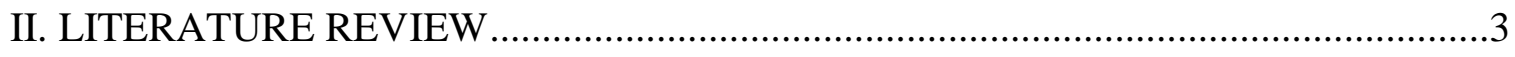

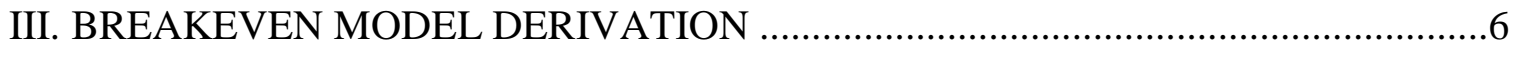

IV. NUMERICAL ANALYSIS OF INITIAL MODEL ................................................11

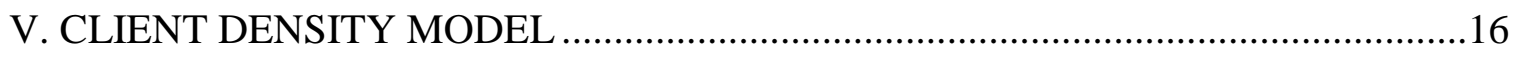

VI. NUMERICAL ANALYSIS OF CLIENT DENSITY MODEL ..................................19

VII. CONCLUSIONS AND FUTURE RESEARCH DIRECTIONS ………………........23

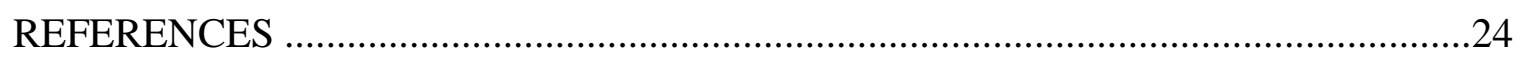

\section{APPENDICES}

A. COORDINATES AND COSTS OF 3PL USAGE FOR EACH CLIENT

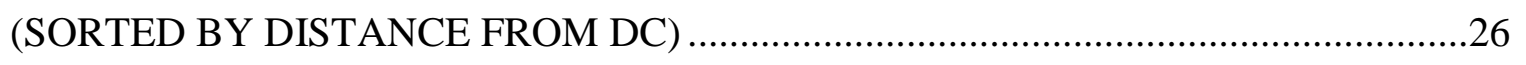

B. COORDINATES OF CLIENT LOCATION FOR NUMERICAL ANALYSIS OF

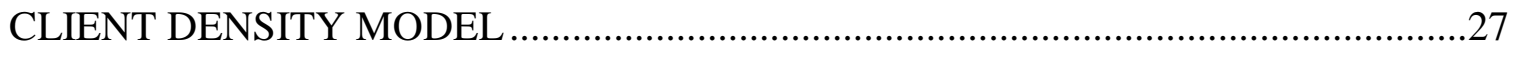




\section{LIST OF TABLES}

Table

Page

1. Notation of parameters and variables used in Chapter III .....................................6

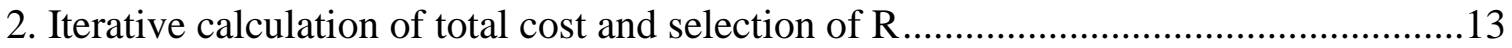

3. Sample ranges for parameters and values for Chapter IV ......................................14

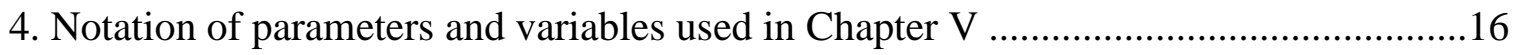

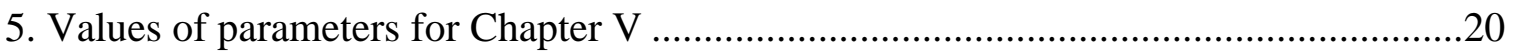




\section{LIST OF FIGURES}

Figure

Page

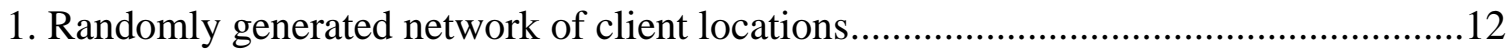

2. Client location with respect to breakeven radius .................................................15

3. Potential for use of in-house distribution based on client density ............................17

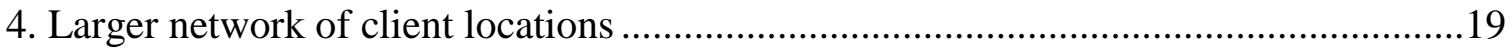

5. Establishing a breakeven radius for the large network ..........................................22

6. Area of consideration outside of breakeven radius ..............................................21 


\section{Introduction}

Large-scale supply chains have never been more expansive than they are today, both functionally within the supply chain and geographically. The measured integration of companies through an agreed-upon division of functions is a large factor in optimizing the supply chain. Customers, external and internal, are stationed across the globe, bolstering the use of international logistics. However, despite the global scale ofmost industries, local distribution is still a vital function of the supply chain.

According to the Council of Supply Chain Management Professionals in its25th Annual State of Logistics Report (published in 2014 for the 2013 fiscal year), $\$ 852$ billion ( $61.5 \%$ of total US logistics costs) can be attributed to transportation costs. Of the total transportation cost, $\$ 657$ billion (47.4\% of total costs, and $77.1 \%$ of transportation costs) were used for truck-based distribution, either intercity or local/regional. Although these high costs may be indicative of a striving economy, they may also be hiding unnecessary spending due to inefficiencies of logistics decisions and systems.

This thesis is motivated by the need of a regional company to select between investing in an in-house logistics system, vesus contracting the services of athird party logistics company., The regional company must calculate which method will be more cost-effective given the particular characteristics of its facilities, inventories, locationspecific costs and conditions, and client distribution. The company may also consider a hybrid logistics system. For example, it may be beneficial for a company to service larger clients using in-house logistics, but outsource to 3PL the service of smaller or remote clients.. In that event, which clients should remain as part of those serviced by in-house and which clients should be moved to third party logistics providers? 
Only cost will be considered in this thesis, since cost is often the most important consideration. Alternative objectives, such as aspects of customer service and environmental impact, are less easily quantified. This thesis develops a breakeven analysis between in-house logistics and third party logistics with the goal of optimizing logistics costs. An analysis of the relationship between the density of clients and the feasibility of using in-house logistics based on distance from a pre-existing distribution center is presented. Both the breakeven analysis and the density analysis are followed by respective numerical analyses based on computer-simulation. Finally, conclusions of this research are presented, and future directions are proposed. 


\section{Literature Review}

This literature review will first note the nearly universal use of third party logistics, guidelines used for outsourcing decision-making, and the impact of third party logistics on company function. Then, the international use of logistics as well as sustainability will be briefly covered, followed by current papers on distribution optimization.

The decision between in-house logistics and outsourcing to third party logistics providers has always been essential to the performance of a firm. Recommendations about logistics were drawn by Wanke and Zinn (2004) with respect to three strategic decisions: push vs. pull inventory deployment, inventory centralization vs. decentralization, and made to order vs. made to stock. These recommendations span many of the criteria used in evaluating outsourcing logistics, including delivery time, perishability, and cost density. Through surveys, Sohail et al. (2006) and Lieb and Bentz (2005) indicated that in three nearly separate economic spheres (Singapore and Malaysia, and America), third-party logistics was on the rise both in terms of company expenditure and breadth of applications within the company. They each analyze the benefits of third party logistics (cost, customer service) and the use of contracts with third party logistics

providers in their respective areas. Sankaran et al. (2002) found out more about the use of third party logistics contracts in New Zealand. Wanke et al. (2007) studied, from the results of a comprehensive survey sent to Brazilian shippers, how these shippers choose between functional and integrated third party logistics services with respect to their manufacturing process structure, as well as the level of sophistication of their existing logistics functions. Grawe (2009) reviewed literature from contemporary publications to 
investigate current innovations in the field of third party logistics, while Bolumole (2001) thoroughly analyzed third-party logistics providers and their impact on a strategic, tactical, and operational level. The wealth of topics and papers about third party logistics pinpoint it as a major player in the logistics field in current years and years to come.

Many of the papers surrounding the topic of third party logistics incorporate a global aspect of supply chain management. Schoenherr (2009) provided an overview of international logistics through the summary of select current publications. A decisionmaking module was developed by Creazza et al. (2010) for logistics network configurations in a global context. Zhu et al. (2002) made a case of "distriparks" (integrated third party logistics provider) from Singapore to emphasize the expansion both of third party logistics and of the scale on which economic activities must be considered. Sustainability is also a huge issue, especially with the discrepancy in the rate of growth of manufacturing industries with their respective supply chains and the rate of growth of freight flow, as is evidenced by the analysis of transportation logistics performed by Rahman et al. (2013).

A few case studies are also available regarding the subject of distribution. Kumar et al. (2006) used goal programming to create multiple feasible solutions for a designated third party logistics allocation problem. Their methodology can be generalized towards the selection of third party logistics providers and allocating services to specific networks. Iannone (2012) analyzed the use of "interports," which can be defined as intermediate nodes in a network of distribution, in Italy in minimizing logistics costs. These papers tend to focus on logistics functions built into the companies, as opposed to combining third party logistics into the decisions. Contesse et al. (2005) used mixed 
integer programming to optimize the profits generated from daily natural gas sales and transportation through pipelines. Facility design and location is also a good variable to scrutinize when it comes to improving supply chain functions; Ulstein et al. (2006) followed Elkem's silicon division's efforts to enhance the efficiency of its supply chain through mathematical modeling.

However, there are few studies on local/regional distribution; the studies mentioned here make cases for the use of third party logistics based on benefits garnered and the effects on the overall supply chain. Despite this, these papers do not explicitly address how to optimize the use of third party logistics in conjunction with in-house functions. The purpose of this thesis is to offer a model for quantitative decision-making that can be used by regional distribution services in order to make logistics decisions more cost-effective. 


\section{Breakeven Model Derivation}

This initial model is concerned with finding a range at which the cost of using third party logistics is equal to that of using in-house logistics. As such, traveling costs are broken down into the parameters seen in Table 1 . These costs are specifically defined and subsequently manipulated to find a mathematical relationship between these traveling costs and inter-client distances, ultimately with the goal of defining the aforementioned range based on the parameters.

Table 1. Notation of parameters and variables used in Chapter III

\begin{tabular}{|c|c|}
\hline \multicolumn{2}{|c|}{ Parameters/Variables } \\
\hline $\begin{array}{l}\mathrm{R}=\text { radius at which using } 3 \mathrm{PL} \text { is equal in } \\
\text { cost to in-house ("breakeven radius") } \\
\mathrm{n}_{\mathrm{p}}=\text { number of clients to be serviced by } \\
\text { method } \mathrm{p} \\
\mathrm{c}=\text { cost per delivery by } 3 \mathrm{PL} \text { (assumed } \\
\text { constant) } \\
c_{i}=\text { cost of delivery to client } \mathrm{i} \text { with in- } \\
\text { house logistics } \\
\mathrm{O}=\text { average overhead cost } \\
\mathrm{p}=\text { price of fuel }\end{array}$ & $\begin{array}{l}\bar{d}=\text { average distance per delivery } \\
\mathrm{M}=\text { unit distance } / \text { unit volume of fuel } \\
\mathrm{w}=\text { wages of driver (currency/unit time) } \\
\bar{t}=\text { average time per delivery } \\
\gamma=\text { unit-less proportionality constant to } \\
\text { relate } \bar{d} \text { and } \mathrm{R} \\
\mathrm{e}=\text { unit-less efficiency factor (based on } \\
\text { volume carried) } \\
\mathrm{V}=\text { volume of product carried }\end{array}$ \\
\hline
\end{tabular}

The derivation of this model assumes that there is a predetermined network of clients, which satisfies any prior conditions (such as having a previous relationship or being a top-paying client), and that there will be one driver servicing the entire network of clients. This model can be generalized to multiple drivers - applied per driver when considering multiple drivers for the same network — and a dynamic network (clients moving in and out of the network) through an application for each instance of time. The accuracy of the model may suffer with deviations from the original assumptions. 
First, define the breakeven radius $\mathrm{R}$ as the distance from a centralized distribution center (DC) such that using third party logistics (3PL) services is financially equal to using an in-house (IH) distribution system. R can be found by satisfying the boundary condition where the costs of these two functions are equal, namely:

$$
\operatorname{cost}_{3 \mathrm{PL}}=\operatorname{cost}_{\mathrm{IH}}
$$

These two costs can be defined by the following:

$$
\operatorname{cost}_{3 P L}=n_{3 P L} c
$$

and

$$
\operatorname{cost}_{\mathrm{IH}}=\sum_{i=1}^{\mathrm{n}_{I H}} c_{i}
$$

The in-house distribution costs per delivery (e.g. assembling and palletizing goods to be shipped) are assumed to be equal between outsourcing to a 3PL service provider and delivering goods with in-house resources. Since overhead is generally measured as a cost per period of time, it can be considered to be a fixed cost in that period of time, whose contribution to the general cost structures can be defined as nO. Thus, the overhead cost per delivery, called $\mathrm{O}$, can be calculated by estimating the total overhead costs (nO) and dividing by the estimated number of deliveries that the resources to be used will be able to cover in the given period.

From the separation of costs into a direct material component, a direct labor component, and an overhead component, the costs incurred by in-house systems per delivery (Eq. 3) can be divided into:

$$
\mathrm{c}_{\mathrm{i}}=\mathrm{c}_{\mathrm{i}, \mathrm{gas}}+\mathrm{c}_{\mathrm{i}, \mathrm{labor}}+\mathrm{O}_{\mathrm{i}}
$$

Using the mathematical definition of the mean, $\overline{c_{l}}=\sum_{i=1}^{n} c_{i} / n$, to define $\sum_{i=1}^{n} c_{i}=$ $\mathrm{n}_{\mathrm{IH}} \overline{\mathrm{C}_{l}}$, Eq. $4 \mathrm{a}$ can be rewritten as the following: 


$$
\overline{c_{l}}=\overline{c_{g a s}}+\overline{c_{\text {labor }}}+\mathrm{O}
$$

Eq. $4 \mathrm{~b}$ uses $\overline{c_{l}}$ as the mean cost per in-house delivery. O, as mentioned above, is fixed across deliveries. Eq. 4b redefines Eq. 3:

$$
\operatorname{cost}_{\mathrm{IH}}=\mathrm{n}_{\mathrm{IH}}\left(\overline{c_{\text {gas }}}+\overline{c_{\text {labor }}}+\mathrm{O}\right)
$$

The average cost of gas $\overline{c_{g a s}}$ can be defined as $p \bar{d} / M$, where $\bar{d}$ is the average distance traveled per delivery (generally the distance between client locations) within $\mathrm{R}$. $\mathrm{M}$ is the unit distance per unit volume of fuel, such as miles per gallon, and $\mathrm{p}$ is the cost of fuel per unit volume. Likewise, the average cost of labor $\overline{c_{\text {labor }}}$ can be defined as $\mathrm{w} \bar{t}$, where $\mathrm{w}$ is the wage of the driver (assumed to be fixed between drivers) and $\bar{t}$ is the average time needed per delivery. With this, Eq. 5 can be written:

$$
\operatorname{cost}_{\mathrm{IH}}=\mathrm{n}_{\mathrm{IH}}\left[\frac{p \bar{d}}{M}+w \bar{t}+O\right]
$$

Reformulating Eq. 1 using the above definitions,

$$
\operatorname{cost}_{\mathrm{IH}}=\mathrm{n}_{\mathrm{IH}}\left[\frac{p \bar{d}}{M}+w \bar{t}+O\right]=\mathrm{n}_{3 \mathrm{PL}} \mathrm{c}=\operatorname{cost}_{3 \mathrm{PL}}
$$

Solving for $\bar{d}$ in Eq. 7a,

$$
\bar{d}=\left(\frac{M}{p}\right)(\mathrm{c}-\mathrm{w} \bar{t}-\mathrm{O})
$$

It is possible to break $\bar{t}$ into a non-value-added component (transportation of the set of goods to its destination) and a value-added component (interaction with the client and replenishment of client's inventory). This can take the form $\bar{t}=(\bar{d} / \bar{v})+\bar{t}_{\mathrm{CS}}$, where $\bar{d}$ is as previously defined, $\bar{v}$ is the average travel velocity between destinations, and $\bar{t}_{\mathrm{CS}}$ is the average time spent in customer service. This is not considered here for the sake of simplicity, though further efficiencies could be achieved from minimizing travel time and maximizing value-added client service time. 
The average distance $\bar{d}$ can be considered to be a function of $\mathrm{R}$, due to the fact that only clients within R are considered in the calculation of $\bar{d}$. It is assumed here that $\bar{d}$ is linearly proportional to R by a unit-less proportionality constant $\gamma$, such that $\bar{d}=\gamma \mathrm{R}$. Within a network of reasonable size, $\gamma$ can be limited to less than or equal to 1 , but greater than 0 ; there are few configurations for which this is not satisfied. Using this relationship and solving for $\mathrm{R}$, Eq. $7 \mathrm{~b}$ becomes

$$
\mathrm{R}=\left(\frac{M}{\gamma p}\right)(\mathrm{c}-\mathrm{w} \bar{t}-\mathrm{O})
$$

It is important to note here that, from the definition of the breakeven radius in Eq. 7a, the cost of in-house logistics can also be less than the cost of using 3PL services and thus all subsequent equations that use this definition can be seen as inequalities. For simplicity, the equations are kept as stated.

The final condition that must be satisfied is capacity efficiency - that is, how much distance is traveled with a partial or empty vehicle load. The distance efficiency factor e ranges in value from $1 / 2$ to 1 . Eq. 8 measures the benefit garnered from the use of in-house logistics instead of outsourcing to third-party logistics providers, as evidenced in the expression $(\mathrm{c}-\mathrm{w} \bar{t}-\mathrm{O})$. For optimal utilization, a situation where goods are flowing both to and from the DC in full vehicle loads, e would take a value of 1 . There is no instance where the vehicle is travelling what can be called "empty miles," where the vehicle is travelling and not carrying a load. In a pure distribution setting (e.g. food delivery, transporting people), empty miles must be travelled from the final client destination back to "home base." On the other hand, if the vehicle makes a trip to every client and returns empty after each trip to the DC, e would take a value of $1 / 2$. For more explicit volume dependence, e can be defined: 


$$
\mathrm{e}=\left(\sum_{i=1}^{n} V_{i}\right) / \mathrm{n}_{I H} V
$$

In Eq. $9 \mathrm{a}, \mathrm{V}$ is used to denote the maximum volume or capacity of the vehicle, whereas $V_{i}$ is used to denote the volume being carried in the vehicle on each leg of delivery to each client. Theoretically, e can be less than $1 \frac{1}{2}$ (worst case -0 ), but these cases are very unlikely. Including this into Eq. $7 \mathrm{~b}$ and 8 , Eq. $9 \mathrm{~b}$ and $9 \mathrm{c}$ follows:

$$
\begin{aligned}
& \bar{d}=\left(\frac{M e}{p}\right)(\mathrm{c}-\mathrm{w} \bar{t}-\mathrm{O}) \\
& \mathrm{R}=\left(\frac{M e}{\gamma p}\right)(\mathrm{c}-\mathrm{w} \bar{t}-\mathrm{O})
\end{aligned}
$$

Continually using the Traveling Salesman Problem to calculate $\bar{d}$ is computationally expensive, especially with increasingly larger networks. Thus, using a pair of chosen parameters, in this case R and gamma (both estimated), can be much more efficient in determining the distribution methods. In the partial absence of estimable parameters, it is possible to choose $\mathrm{R}$ and decide whether the remaining unknown parameters are of acceptable magnitude. For example, for large $\mathrm{R}$ and small $\mathrm{M}$ and $\mathrm{p}$ (both near 1), the cost of using 3PL would have to be high, with drivers' wages and overhead being low values relative to that cost. This will be numerically explored in the next chapter. 


\section{Numerical Analysis of Initial Model}

From both a theoretical and practical standpoint, $\mathrm{R}$ can be (less than or) equal to 0 - for $\mathrm{R} \leq 0$, c must be less than or equal to the expression $(\mathrm{w} \bar{t}+\mathrm{O})$. No matter which factor dominates, as long as the addition of the wage of the driver and the overall overhead costs surpasses the value of the cost of using 3PL, R will be less than 0 . This has no practical application; for this entire case, $\mathrm{R}$ can just be considered to be 0 , meaning that for all clients in the network, 3PL is the more cost-effective method for local distribution. It is prudent to remember that the parameters mentioned here are considered to be averages and that therefore there may be some clients that fulfill the cost efficiency requirement for the usage of in-house logistics.

One method by which to optimize $\mathrm{R}$ is to iteratively find an acceptable value of $\mathrm{R}$. This would involve alternating between calculating the total cost of distribution and increasing the radius to include one additional client into the network to be serviced by in-house logistics. As mentioned in the previous chapter, in the face of inestimable parameters (or unsteady parameters, which violates the key assumption of constant 3PL costs), this process would be painstaking, but the most thorough in finding the ideal balance between the two distribution systems. If all the assumptions of the model are met, a highly improbable situation, then the model should theoretically find a suitable breakeven radius.

To illustrate the iterative process, a network of points representing clients will be generated via Excel's (pseudo)random function. A base number for both $\mathrm{x}$ and $\mathrm{y}$ coordinates are generated using common angles $\left(30^{\circ}, 60^{\circ}\right.$, etc.) from a circle of radius 10 ; to avoid ambiguity of which quadrant in which this point will fall, no points with base 
numbers on an axis $(\mathrm{x}=0$ or $\mathrm{y}=0)$ are used. Then random values, either positive or negative and with absolute value less than 5 , are generated and then summed with the base numbers of $\mathrm{x}$ and $\mathrm{y}$. To round off the set to a total of 20 points, 4 additional points are made purely from the smaller random values that are multiplied by 5 rather than the full radius of 10. An example set can be seen in Figure 1 below.

\section{Random Client Locations}

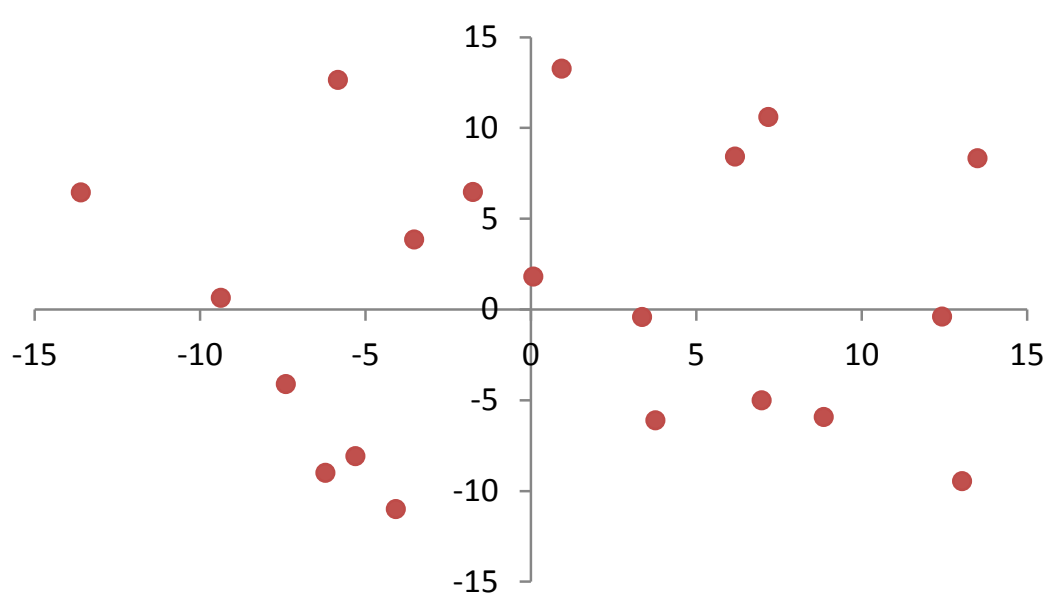

Fig. 1. Randomly generated network of client locations

The costs of the in-house distribution method will be calculated through Eq. 7a, whereas the cost of 3PL distribution will take the form of Eq. 3 for the sake of varying costs between clients. For this numerical analysis, $\mathrm{M}$ will be 5 miles/gallon, $\mathrm{p}$ will be $\$ 4 /$ gallon, w will be $\$ 0.40 /$ minute, $\bar{t}$ will be 50 minutes, $\mathrm{O}$ will be $\$ 500$, and c will range from $\$ 100$ to $\$ 1000$ (again generated at random and rounded to the nearest 10 ). See Appendix A for values of c, as well as coordinates of clients in Figure 1. All values here for the parameters will be arbitrary, except for $\mathrm{n}$ (number of clients within the breakeven radius) and $\bar{d}$ (average distance between those clients). $\mathrm{n}$ will be chosen, and $\bar{d}$ is to be 
calculated, through each iteration. The calculations of costs at various radii (denoted by the number of clients) can be seen in Table 2.

Table 2. Iterative calculation of total cost and selection of $\mathrm{R}$

\begin{tabular}{|l|l|l|l|}
\hline $\begin{array}{l}\text { Number of clients } \\
\text { within R }\end{array}$ & $\begin{array}{l}\text { Total cost of in-house } \\
\text { logistics }\end{array}$ & $\begin{array}{l}\text { Total cost of 3PL } \\
\text { distribution }\end{array}$ & $\begin{array}{l}\text { Total cost of } \\
\text { distribution }\end{array}$ \\
\hline 0 & $\$ 0$ & $\$ 11,390$ & $\$ 11,390$ \\
\hline 1 & $\$ 522.88$ & $\$ 10,550$ & $\$ 11,072.88$ \\
\hline 5 & $\$ 2,630$ & $\$ 9,410$ & $\$ 12,040.24$ \\
\hline 10 & $\$ 5,251.59$ & $\$ 5,530$ & $\$ 10,781.59$ \\
\hline 15 & $\$ 7,863.26$ & $\$ 2,030$ & $\$ 9,893.26$ \\
\hline 19 & $\$ 9,974.46$ & $\$ 310$ & $\$ 10,284.46$ \\
\hline 20 & $\$ 10,478.77$ & $\$ 0$ & $\$ 10,478.77$ \\
\hline
\end{tabular}

From the above table, the minimum total cost seems to be associated with distributing to 15 clients with in-house logistics and 5 clients with 3PL; this holds true for all points in the mock data. In a more general sense, the model can be used to find the most cost-efficient blend of in-house logistics and 3PL; with larger networks, this iterative process is much more complex, and equation 9c should provide the breakeven radius with less work.

However, due to how small the random numbers involved are for the coordinates (as well as the wages), this total cost mainly reflects the difference in values between the high overhead cost per client and the cost of using 3PL. If the distances are increased by an order of magnitude, both of these seemingly insignificant factors could prove to outweigh the overhead in more practical situations. 
Starting again from the clients shown in Figure 1, the breakeven radius is to be generated via estimates of the parameters mentioned. The parameters, as well as minimums and maximums for each, can be seen in Table 3. Minimum and maximum values for the efficiency factor (e) and the proportionality constant $(\gamma)$ are as discussed in the previous chapter. Minimum and maximum values for $\mathrm{M}, \mathrm{p}$, and $\mathrm{w}$ are provided courtesy of Oak Ridge National Laboratory (Center of Transportation Analysis), US Energy Information Administration, and US Dept. of Labor (Bureau of Labor Statistics), respectively. Without accurate data on the cost of distribution via 3PL (c), the time spent by a driver with each client $(\bar{t})$, or the overhead cost per client $(\mathrm{O})$, these values are much more arbitrary.

Table 3. Sample ranges for parameters and values for Chapter IV

\begin{tabular}{|l|l|l|l|}
\hline Parameters & Minimum & Maximum & Value \\
\hline $\mathrm{M}$ (in mpg) & 5 & 25 & 5 \\
\hline $\mathrm{e}$ & 0.5 & 1 & 1 \\
\hline$\gamma$ & 0 & 1 & 1 \\
\hline $\mathrm{p}$ (in USD/gallon) & 2.5 & 10 & 4 \\
\hline $\mathrm{c}$ (in USD) & 100 & 10000 & 528 \\
\hline $\mathrm{w}$ (in USD/min) & 0.2167 & 0.45 & 0.4 \\
\hline $\bar{t}$ (in min) & 5 & 50 & 50 \\
\hline O (in USD) & 50 & 5000 & 500 \\
\hline
\end{tabular}

For Figure 2, the values of the parameters have been specifically chosen to obtain a breakeven radius of 10 (miles) from the previously listed values. 


\section{Client location vs. Radius}

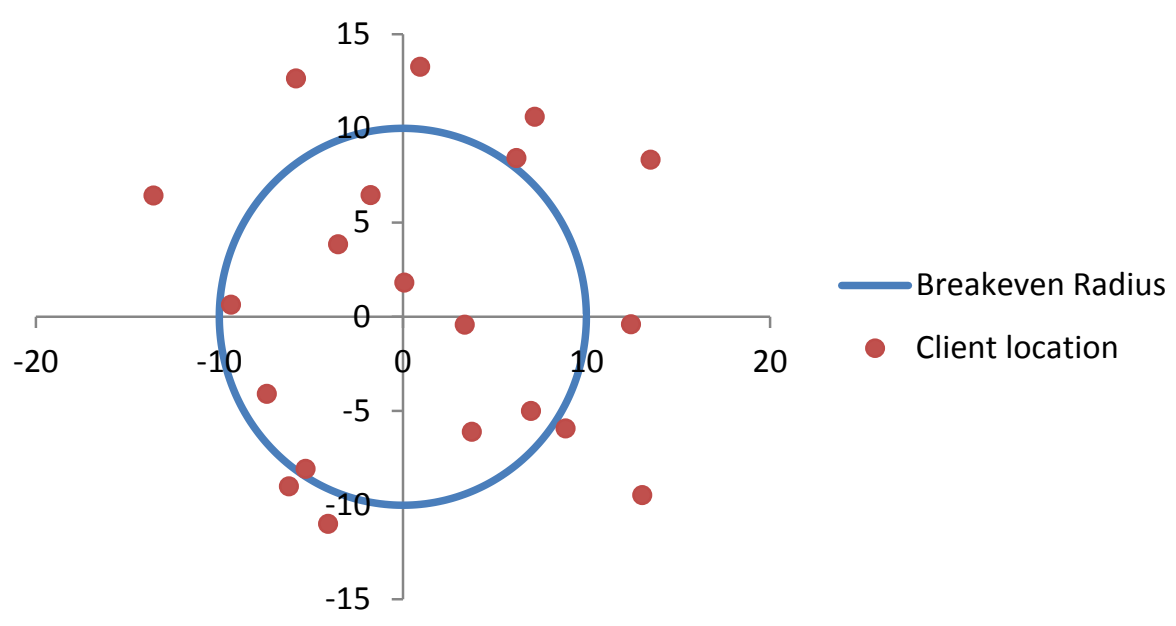

Fig. 2. Client location with respect to breakeven radius

In Figure 2, there are 9 clients within $R=10$, with 2 clients between $R=9$ and $R$ $=10$, and there are 3 additional clients between $\mathrm{R}=10$ and $\mathrm{R}=11$. With a more realistic efficiency factor such as 0.65 and higher fuel efficiency such as 10 , the breakeven radius increases drastically to 13 , which contains 15 clients. It is advisable to keep in mind that percentage differences in the first four parameters are multiplicative to the breakeven radius, while differences in last four parameters are generally magnified in their effect. The clients on which to keep an eye are, of course, those near the extent of the breakeven radius, as small fluctuations in the multiplicative parameters or significant differences in the parameters in the multiplied expression could cause the cost-optimal distribution method to waver. In this situation, it would be best to investigate other factors that are not strictly cost-based to determine a distribution method for these clients. 


\section{Client Density Model Derivation}

The client density model builds off of the initial model, using the same equations and basing its results on those of the initial model; thus, the derivation of this model is much shorter than that of the initial model. In effect, the client density model seeks to reform the logistics decisions made for clients outside the breakeven radius based on proximity to other clients.

Table 4. Notation of parameters and variables used in Chapter V

\begin{tabular}{|l|l|}
\hline \multicolumn{2}{|c|}{ Parameters/Variables } \\
\hline $\mathrm{R}=$ breakeven radius centered on DC & $\mathrm{c}=$ cost per delivery by 3PL (assumed \\
$\mathrm{D}=$ distance between DC and center of & constant) \\
external area & $\mathrm{w}=$ wages of driver (currency/unit time) \\
$\mathrm{r}=$ breakeven radius of area of & $\bar{t}=$ average time per delivery \\
consideration & $\gamma=$ unit-less proportionality constant to \\
$\bar{d}_{\text {within }}$ average distance per delivery & relate $\bar{d}$ and $\mathrm{R}$ \\
$\mathrm{M}=$ unit distance / unit volume of fuel & $\mathrm{e}=$ unit-less efficiency factor (based on \\
$\mathrm{p}=$ price of fuel & volume carried) \\
$\mathrm{n}_{\mathrm{r}}=$ number of clients within the area of & $\eta=$ client density in external area \\
consideration & \\
\hline
\end{tabular}

In Chapter III, $\bar{d}$ was defined as the average distance between clients within $\mathrm{R}$. In the scenario presented in this chapter, this parallels only the average distance between clients within a circular area centered outside the initial area covered by in-house logistics. Figure 3 at the top of the next page shows the new designation of spaces, the initial area centered on the DC with radius $\mathrm{R}$ and the external area-referred to as the area of consideration - with radius $r$. The distance between the DC and the center of this external area will be designated as D. 


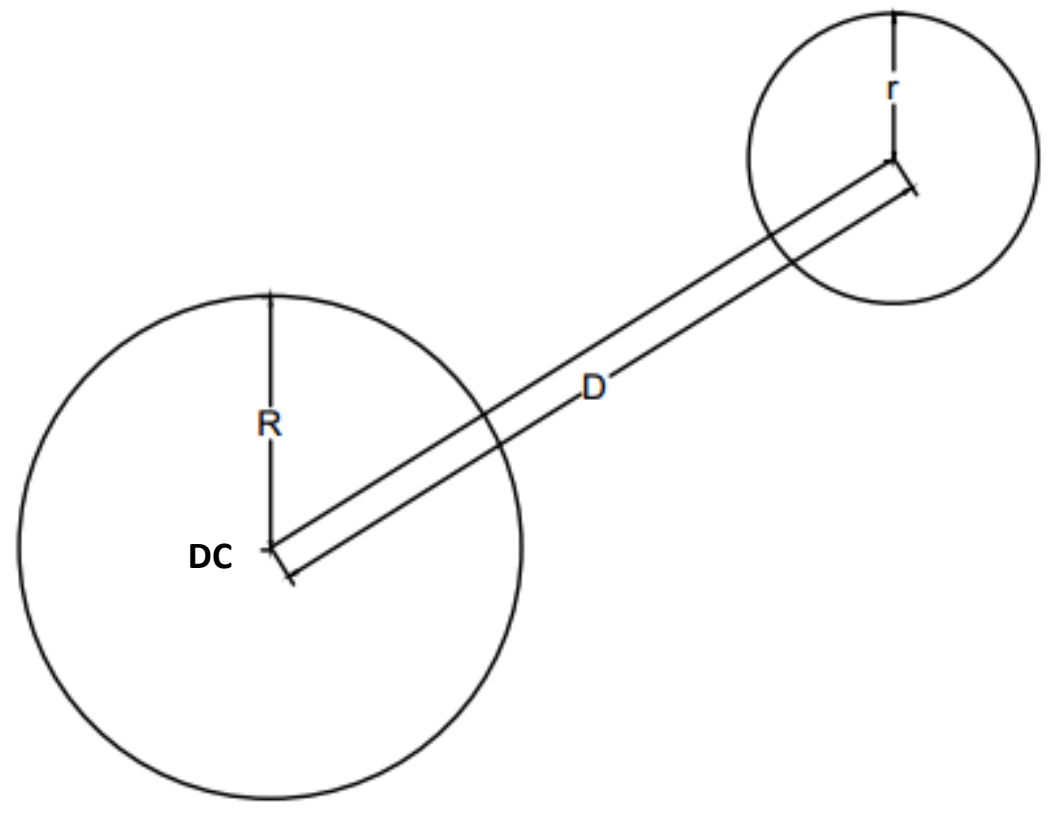

Fig. 3. Potential for use of in-house distribution based on client density

Eq. 9b (stated below), while useful, needs to be modified to account for this extra distance instead of simply calculating the average distance within the external area.

$$
\bar{d}_{\text {within }}=\left(\frac{M e}{p}\right)(\mathrm{c}-\mathrm{w} \bar{t}-\mathrm{O})
$$

The average distance that a driver must travel per delivery $\bar{d}$ for clients in this external area is the addition of the term brought in by Eq. $7 \mathrm{~b}$ and an extra term for D, which symbolically is $\bar{d}=\bar{d}_{\text {within }}+\frac{2 D}{n_{r}}$. Eq. 10 is the result:

$$
\bar{d}=\left(\frac{M e}{p}\right)(\mathrm{c}-\mathrm{w} \bar{t}-\mathrm{O})+\left(\frac{2 D}{n_{r}}\right)
$$

Using the same proportionality method as in Chapter III , $\bar{d}=\gamma \mathrm{R}$, and the reworked definitions introduced in this chapter, Eq. 11a and 11b will continue the derivation of the client density model. 


$$
\gamma r=\left(\frac{M e}{p}\right)(\mathrm{c}-\mathrm{w} \bar{t}-\mathrm{O})+\left(\frac{2 D}{n_{r}}\right)
$$

Moving constants around,

$$
n_{r} r=\left(\frac{M n_{r} e}{\gamma p}\right)(\mathrm{c}-\mathrm{w} \bar{t}-\mathrm{O})+\left(\frac{2 D}{\gamma}\right)
$$

To solve for the client density $\eta=n_{r} / r^{2}$,

$$
\eta=\left(\frac{M n_{r} e}{\gamma p r^{3}}\right)(\mathrm{c}-\mathrm{w} \bar{t}-\mathrm{O})+\left(\frac{2 D}{\gamma r^{3}}\right)
$$

Aside from the terms carried over from the basic terms from Eq. 9b, there remains the $n_{r} / r^{3}$ factor on the first expression, which describes the relative efficiency of using in-house distribution over 3PL, and the $\frac{D}{\gamma r^{3}}$ term, which describes the tradeoff between the distance from the DC to the area of consideration and having a large area of consideration. The former, due to the $n_{r} / r^{3}$ factor noted previously, scales nearly identically to $\eta$ itself - the benefit of using in-house logistics scales linearly with the number of clients being serviced, but it scales exponentially with the inverse of the radius of the area of consideration. As expected, the latter forces the optimal client density to be large for large $\mathrm{D}$, while adding only a small value for large $\mathrm{r}$ to an already small $\eta$. From an analytical standpoint, the optimal client density cannot be accurately gauged; therefore, another numerical analysis is necessary. 
VI. Numerical Analysis of Client Density Model

Similar to the methodology shown in Chapter IV, 100 points are generated to simulate client location distributed around the DC; 80 of these points are generated from the sum of base $\mathrm{x}$ - and $\mathrm{y}$-coordinates and random values, while the remaining 20 are generated from the sum of two sets of random values. The base coordinates are derived from the same angles of circles of various radii $(10,20,30,40,50-16$ pairs of coordinates per radius), and the random values are from Excel's rand() function multiplied by half the radius (4 pairs of coordinates per radius). The list of points, with the first 20 points taken directly from the data seen in Chapter IV, can be found in Appendix B; the points are graphically represented in Figure 4 below.

\section{Client Locations}

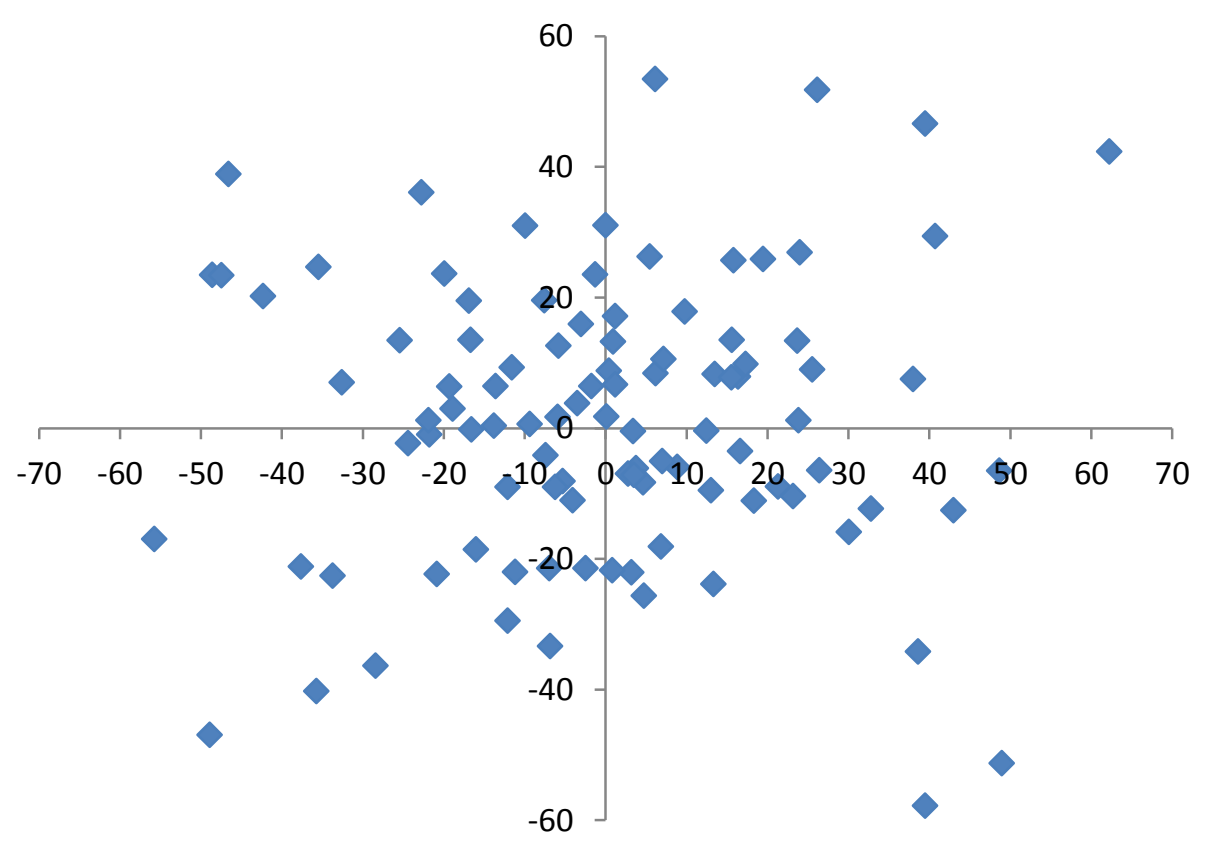

Fig. 4. Larger network of client locations

Using different parameters from Chapter IV (see Table 5 on next page), a breakeven radius that does not include every single client is established. 
Table 5. Values of parameters for Chapter V

\begin{tabular}{|l|l|}
\hline Parameter & Value \\
\hline $\mathrm{M}$ (in miles/gallon) & 8 \\
\hline$\gamma$ & 0.6 \\
\hline $\mathrm{p}$ (in USD/gallon) & 1 \\
\hline $\mathrm{c}$ (in USD) & 6 \\
\hline $\mathrm{w}$ (in USD/min) & 350 \\
\hline $\bar{t}$ (in min) & 0.45 \\
\hline O (in USD) & 50 \\
\hline
\end{tabular}

This yields a breakeven radius of 22 miles, which contains 48 of the 100 clients to be considered (see Figure 5).

\section{Client Location and Breakeven Radius}

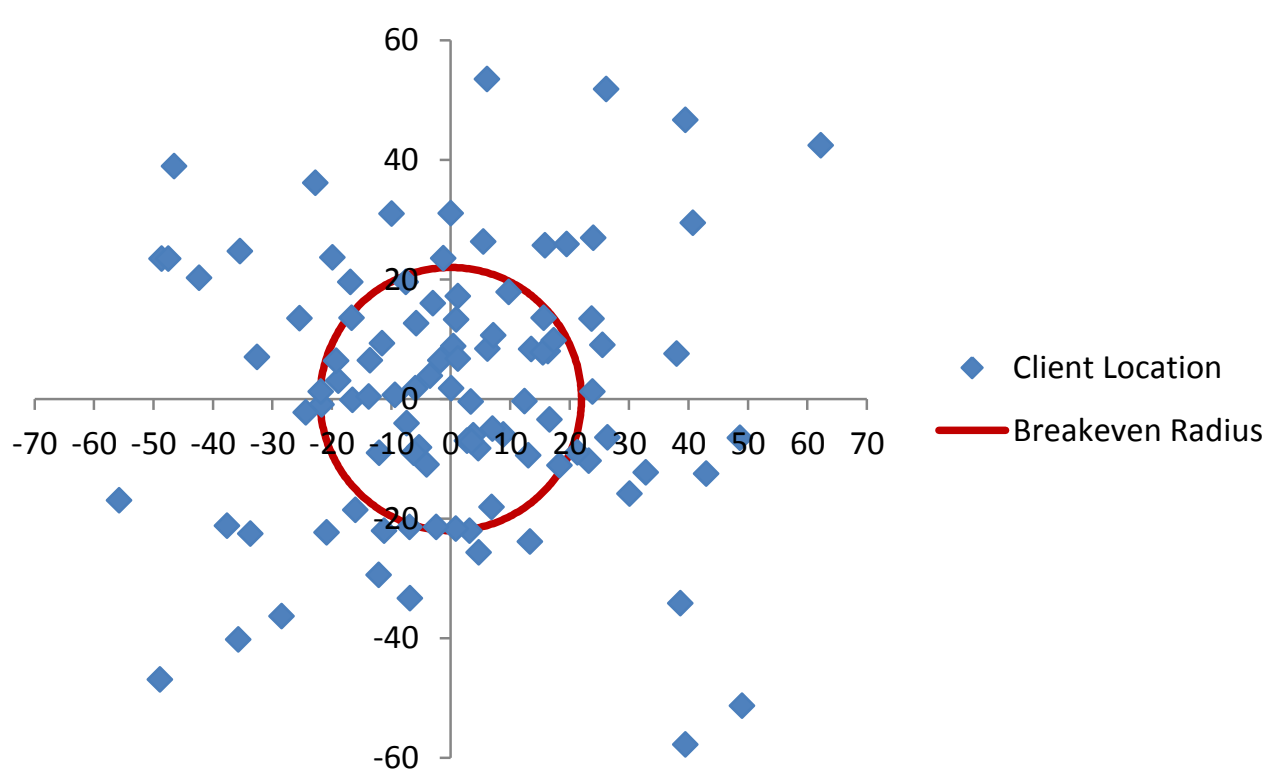

Fig. 5. Establishing a breakeven radius for the large network 
With the breakeven radius established, an area of consideration in which to use inhouse distribution is sought after. Ideally, a matrix would be formed detailing the distance between each and every client, and from that matrix, each subset of clients with adequately small distances would be considered for in-house distribution. In this case, with such a large network mapped out, an area of consideration will simply be chosen from the clusters of clients outside the breakeven radius.

There is a simple method for analyzing whether an area of consideration qualifies for in-house distribution: to select a subset of clients, centering the area between the clients and selecting a radius such that the farthest client lies on the radius, and evaluate whether the density of the clients selected is greater than the expected density with the expected parameters (the right-hand side of Eq. 12). This method is an iterative process in optimizing similar to the one used in Chapter IV.

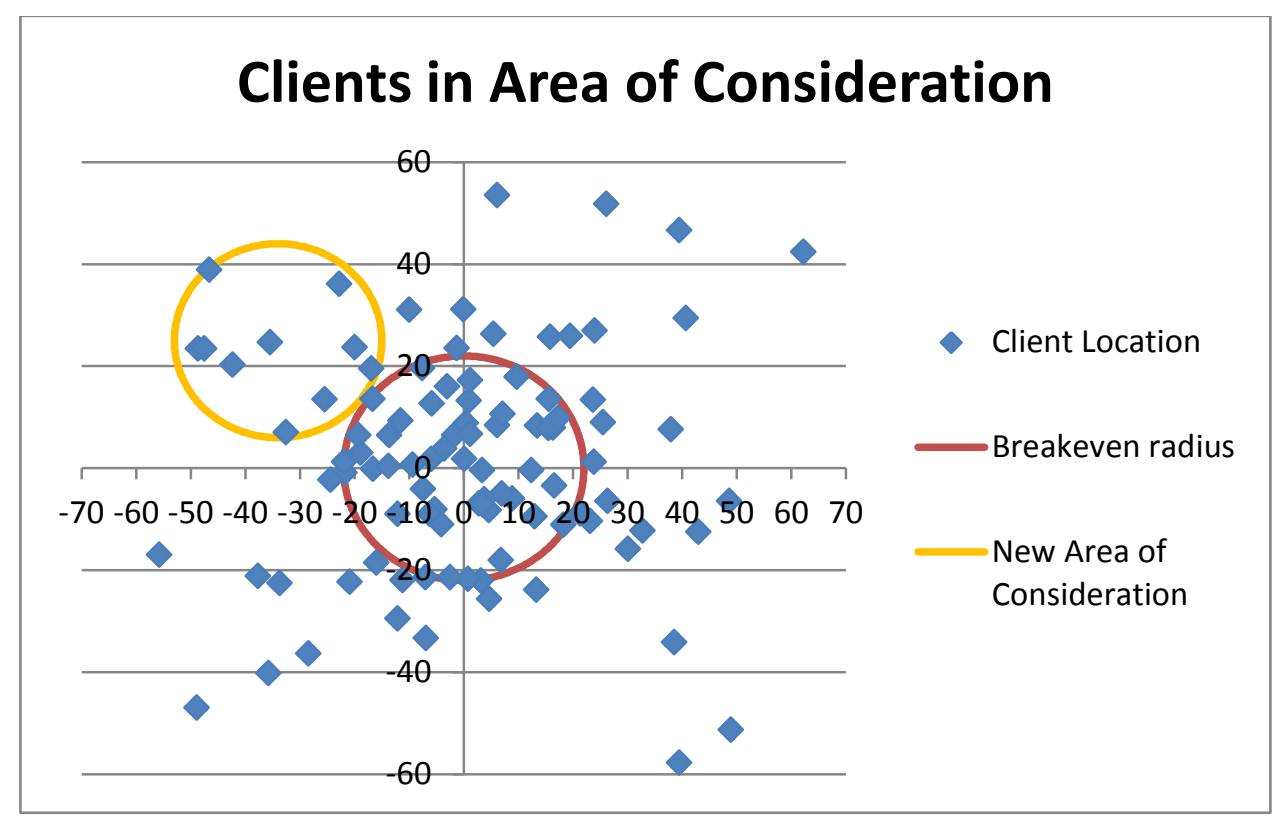

Fig. 6. Area of consideration outside of breakeven radius

With $r=19$ miles and $n=10$ included with the other parameters, the expected density can be calculated to be 0.044 clients per square mile, whereas $\eta$ is 0.0277 clients 
per square mile. As noted in Chapter III, these equations can be viewed as inequalities rather than strict definitive equations, and this is where that evaluation can be of use. By varying the radius of the area of consideration as described above, various $\eta$ values can be obtained and then compared to the calculated right-hand side of Eq. 12. Should the $\eta$ value be larger than the density designated from the expected parameters, then that area of consideration can be serviced with in-house logistics. Otherwise, if no $\eta$ value satisfies this statement, then all clients outside the breakeven radius should be serviced with 3PL.

Analytically, a value of $\eta$ can be considered to be infinite when considering a single client location $(n=1, r=0)$. This also result in an expected density of infinite value and necessarily be undefined as an (in)equality. In practical situations, this would mean a low efficiency for delivery in the case of in-house logistics and is definitely inviable. Where there are large values for $\eta$ for small $n$ values in conjunction with small $r$ values, computing the total cost of distribution for the area of consideration is not computationally expensive and can be done to make the comparison.

In the event of overlap in area between the area of consideration and the original breakeven radius, any clients within that area should be serviced with in-house distribution methods; however, any clients should also be discounted from the area of consideration, decreasing the client density within that area and potentially removing the area of consideration from feasibility. 


\section{Conclusions And Future Research Directions}

This thesis proposed a method to determine the optimal regional distribution method, selecting between in-house logistics and 3PL services in order to reduce direct distribution costs. Starting from the concept of in-house costs vs. cost of 3PL services, a "breakeven radius" was established and clients were assigned to either in-house logistics or 3PL based on relative location to the radius. In this way, the system considered client location to select an optimal method and then analyzed the effect on density of client location to re-evaluate the previously selected optimal distribution method.

From the managerial perspective, most of the parameters discussed here are derived from previous actions (e.g. type of delivery vehicle purchased, agreement to 3PL service cost) and are not directly controlled at the point of logistics decision making.

Further investigations could probe into more efficient methods to analyze client density and its effects on decision-making with regards to distribution and techniques that include other aspects of distribution (e.g. lead time, perishability, integration of services). Another topic that may deserve some analysis is the projected cost-benefit timeline of implementing an in-house logistics system as well as setting up a relationship with one or more 3PL service provider. 


\section{REFERENCES}

Bolumole, Yemisi. “The Supply Chain Role of Third-Party Logistics Providers.” The International Journal of Logistics Management 12.2 (2001): 87-102. ABI/Inform. Web. 30 September 2014.

Contesse, Luis, Juan Carlos Ferrer, and Sergio Maturana. "A Mixed-Integer Programming Model for Gas Purchase and Transportation." Annals of Operations Research 139 (2005): 39-63. ABI/Inform. Web. 6 June 2014.

Creazza, A., F. Dallari, and M. Melacini. "Evaluating Logistics Network Configurations for a Global Supply Chain." Supply Chain Management: An International Journal 15.2 (2010): 154-64. ABI/Inform. Web. 22 May 2014.

Grawe, Scott J. "Logistics Innovation: A Literature-based Conceptual Framework." The International Journal of Logistics Management 20.3 (2009): 360-77. ABI/Inform. Web. 22 May 2014.

Iannone, Fedele. "A Model Optimizing the Port-hinterland Logistics of Containers: The Case of the Campania Region in Southern Italy." Maritime Economics \& Logistics 14.1 (2012): 33-72. ABI/Inform. Web. 22 May 2014.

Kumar, Manoj, Prem Vrat, and Ravi Shankar. "A Multi-objective 3PL Allocation Problem for Fish Distribution." International Journal of Physical Distribution \& Logistics Management 36.9 (2006): 702-15. ABI/Inform. Web. 22 May 2014.

Lieb, Robert, and Brooks A. Bentz. "The Use of Third-Party Logistics Services by Large American Manufacturers: The 2004 Survey." Transportation Journal 44.2 (2005): 515. JSTOR. Web. 30 May 2014.

Rahman, Mohammad, Faisal Mallum, and Chad Miller. "A Sustainability Analysis between Transportation Logistics and Global Trade." Proceedings of the 2013 Industrial and Systems Engineering Research Conference (n.d.): n. pag. ABI/INFORM. Web. 22 May 2014. 
Sankaran, Jay, David Mun, and Zane Charman. "Effective Logistics Outsourcing in New Zealand: An Inductive Empirical Investigation." International Journal of Physical Distribution \& Logistics Management 32.8 (2002): 682-702. ABI/Inform. Web. 22 May 2014.

Schoenherr, Tobias. "Logistics and Supply Chain Management Applications Within a Global Context: An Overview." Journal of Business Logistics 30.2 (2009): 1-25. ABI/Inform. Web. 22 May 2014.

Schulz, John D. " $25^{\text {th }}$ Annual State of Logistics: It's Complicated." Logistics Management. N.p., 01 July 2014. Web. 10 Apr 2015

Sohail, M. Sadiq, Rohit Bhatnagar, and Amrik S. Sohal. "A Comparative Study on the Use of Third Party Logistics Services by Singaporean and Malaysian Firms." International Journal of Physical Distribution \& Logistics Management 36.9 (2006): 690701. ABI/INFORM. Web. 20 May 2014.

Ulstein, Nina Linn, Marielle Christiansen, Roar Grønhaug, Nick Magnussen, and Marius M. Solomon. "Elkem Uses Optimization in Redesigning Its Supply Chain." Interfaces 36.4 (2006): 314-25. JSTOR. Web. 21 Mar. 2014.

Wanke, Peter F., and Walter Zinn. "Strategic Logistics Decision Making." International Journal of Physical Distribution \& Logistics Management 34.6 (2004): 46678. ABI/INFORM. Web. 22 May 2014.

Wanke, Peter, Rebecca Arkader, and Maria F. Hijjar. "Logistics Sophistication, Manufacturing Segments, and the Choice of Logistics Providers."International Journal of Operations \& Production Management 27.5 (2007): 542-59. ABI/Inform. Web. 22 May 2014.

Zhu, Jieming, Han Swee Lean, and Seah Kiat Ying. "The Third-Party Logistics Services and Globalization of Manufacturing." International Planning Studies 7.1 (2002): 89104. ABI/Inform. Web. 22 May 2014. 


\section{APPENDICES}

Appendix A.

Coordinates and costs of 3PL usage for each client (sorted by distance from DC)

\begin{tabular}{|c|c|c|c|c|}
\hline Client \# & X-Coordinate & Y-Coordinate & Distance from DC & 3PL cost \\
\hline 1 & 0.092709 & 1.79449 & 1.796883 & 840 \\
\hline 2 & 3.379595 & -0.42054 & 3.40566 & 360 \\
\hline 3 & -3.51617 & 3.840231 & 5.206809 & 140 \\
\hline 4 & -1.743 & 6.452263 & 6.683543 & 170 \\
\hline 5 & 3.770971 & -6.12088 & 7.189253 & 470 \\
\hline 6 & -7.39481 & -4.11046 & 8.460448 & 720 \\
\hline 7 & 6.987079 & -5.00978 & 8.597511 & 680 \\
\hline 8 & -9.36251 & 0.632402 & 9.383839 & 840 \\
\hline 9 & -5.28833 & -8.0876 & 9.663109 & 920 \\
\hline 10 & 6.188627 & 8.414619 & 10.44533 & 720 \\
\hline 11 & 8.869489 & -5.94344 & 10.67672 & 370 \\
\hline 12 & -6.19944 & -9.0163 & 10.94197 & 700 \\
\hline 13 & -4.06788 & -11.0049 & 11.7327 & 600 \\
\hline 14 & 12.4408 & -0.39732 & 12.44714 & 960 \\
\hline 15 & 7.184734 & 10.59683 & 12.80286 & 870 \\
\hline 16 & 0.940768 & 13.25134 & 13.28469 & 120 \\
\hline 17 & -5.81469 & 12.62821 & 13.90261 & 290 \\
\hline 18 & -13.5882 & 6.42956 & 15.0326 & 780 \\
\hline 19 & 13.51197 & 8.320699 & 15.86844 & 530 \\
\hline 20 & 13.04464 & -9.46945 & 16.11934 & 310 \\
\hline
\end{tabular}


Appendix B.

Coordinates of client location for numerical analysis of client density model

\begin{tabular}{|c|c|c|c|c|c|}
\hline Client \# & X-Coord & Y-Coord & Client \# & X-Coord & Y-Coord \\
\hline 1 & 13.51197 & 8.320699 & 51 & -6.86076 & -33.3201 \\
\hline 2 & 0.940768 & 13.25134 & 52 & -20.8581 & -22.2943 \\
\hline 3 & -5.81469 & 12.62821 & 53 & 3.183015 & -22.078 \\
\hline 4 & -9.36251 & 0.632402 & 54 & 18.31307 & -11.1065 \\
\hline 5 & -7.39481 & -4.11046 & 55 & 26.39589 & -6.46637 \\
\hline 6 & -5.28833 & -8.0876 & 56 & 21.33382 & -8.95133 \\
\hline 7 & 8.869489 & -5.94344 & 57 & 0.821814 & -21.7152 \\
\hline 8 & 13.04464 & -9.46945 & 58 & -16.6559 & 13.53809 \\
\hline 9 & -1.743 & 6.452263 & 59 & 1.181883 & 17.18651 \\
\hline 10 & 3.770971 & -6.12088 & 60 & 4.663171 & -8.26134 \\
\hline 11 & 6.188627 & 8.414619 & 61 & 23.8327 & 1.208126 \\
\hline 12 & 7.184734 & 10.59683 & 62 & 15.5337 & 7.784507 \\
\hline 13 & -3.51617 & 3.840231 & 63 & 26.16045 & 51.76092 \\
\hline 14 & -13.5882 & 6.42956 & 64 & 6.127615 & 53.43936 \\
\hline 15 & -6.19944 & -9.0163 & 65 & -7.5554 & 19.55803 \\
\hline 16 & -4.06788 & -11.0049 & 66 & -35.4663 & 24.68581 \\
\hline 17 & 6.987079 & -5.00978 & 67 & -22.7473 & 36.08657 \\
\hline 18 & 12.4408 & -0.39732 & 68 & -32.5606 & 7.012276 \\
\hline 19 & 0.092709 & 1.79449 & 69 & -37.6509 & -21.1815 \\
\hline 20 & 3.379595 & -0.42054 & 70 & -16.0112 & -18.5589 \\
\hline 21 & 16.36669 & 7.94224 & 71 & -12.0879 & -29.4617 \\
\hline 22 & 9.773314 & 17.88094 & 72 & -28.422 & -36.3562 \\
\hline
\end{tabular}




\begin{tabular}{|c|c|c|c|c|c|}
\hline 23 & 15.62673 & 13.55309 & 73 & 30.09039 & -15.8557 \\
\hline 24 & 15.81821 & 25.67847 & 74 & 13.35516 & -23.8665 \\
\hline 25 & -19.9136 & 23.67441 & 75 & 43.00589 & -12.5348 \\
\hline 26 & -3.00705 & 15.96459 & 76 & 48.63343 & -6.49636 \\
\hline 27 & -18.8969 & 3.011078 & 77 & -16.889 & 19.50601 \\
\hline 28 & -19.2857 & 6.401212 & 78 & 17.291 & 9.80791 \\
\hline 29 & -21.7506 & -0.93798 & 79 & 25.52457 & 8.99202 \\
\hline 30 & -12.0783 & -8.99654 & 80 & -13.7651 & 0.361207 \\
\hline 31 & -6.95589 & -21.4119 & 81 & 62.24315 & 42.36588 \\
\hline 32 & -11.1746 & -22.0169 & 82 & 24.01711 & 26.9177 \\
\hline 33 & 4.720556 & -25.6369 & 83 & 5.457046 & 26.29189 \\
\hline 34 & 6.856723 & -18.0812 & 84 & 39.47987 & 46.64864 \\
\hline 35 & 16.63335 & -3.47146 & 85 & -48.6095 & 23.45984 \\
\hline 36 & 23.17798 & -10.3797 & 86 & -46.5636 & 38.8932 \\
\hline 37 & 0.424842 & 8.794408 & 87 & -47.4804 & 23.42201 \\
\hline 38 & 2.771956 & -6.97327 & 88 & -42.3296 & 20.24647 \\
\hline 39 & 1.172854 & 6.71341 & 89 & -48.884 & -46.9507 \\
\hline 40 & -16.5533 & -0.1606 & 90 & -55.7592 & -16.9725 \\
\hline 41 & 38.00109 & 7.545987 & 91 & -35.7089 & -40.2222 \\
\hline 42 & 40.7196 & 29.41619 & 92 & -2.45848 & -21.4017 \\
\hline 43 & 23.70985 & 13.39408 & 93 & 39.48901 & -57.786 \\
\hline 44 & 19.47745 & 25.89247 & 94 & 48.98492 & -51.2862 \\
\hline 45 & -0.0077 & 31.06204 & 95 & 32.79089 & -12.2954 \\
\hline 46 & -9.93557 & 30.98973 & 96 & 38.61525 & -34.1907 \\
\hline
\end{tabular}




\begin{tabular}{|r|r|r|r|r|r|r|}
\hline 47 & -25.4106 & 13.47807 & & 97 & -1.25856 & 23.50583 \\
\hline 48 & -21.8568 & 1.231076 & & 98 & -5.9169 & 1.742658 \\
\hline 49 & -24.3877 & -2.29788 & & 99 & 3.490399 & -7.12691 \\
\hline 50 & -33.7109 & -22.5508 & & 100 & -11.5684 & 9.322782 \\
\hline
\end{tabular}

